\title{
A Compactness Criterion for Real Plane Algebraic Curves
}

\author{
John Stalker
}

August 1, 2018

\begin{abstract}
Two sets of conditions are presented for the compactness of a real plane algebraic curve, one sufficient and one necessary, in terms of the Newton polygon of the defining polynomial.
\end{abstract}

\section{Introduction}

We do not, at present, have a complete understanding of the possible topologies of real affine plane algebraic curves of given degree. Indeed, this is one half of Hilbert's 16th problem. For any given curve the problem is much easier, but is still complicated in comparison with the complex case. A summary of the current state of knowledge, and some new results, may be found in [1].

This paper is concerned exclusively with compactness of such curves. It is not hard to see that a real affine plane algebraic curve is compact if and only if its points on the line at infinity are all isolated. There are finitely many such points and there exists an effective procedure to check whether they are isolated [3]. That procedure is complicated both to describe and perform, however.

This paper presents a simple necessary criterion and a simple sufficient condition, both of which can be stated in terms of the Newton polygon of the defining polynomial. Unfortunately any criterion which is both necessary and sufficient will not be simple. For almost all curves with a given Newton polygon, however, either the necessary condition fails or the sufficient condition holds. Only for a set of curves of codimension at least one do we need to use the more complicated machinery of $\underline{3}$.

In this paper

$$
p(x, y)=\sum c_{k, l} x^{k} y^{l}
$$

is a polynomial in two variables with real coefficients and $C$ is the curve

$$
C=\left\{(x, y) \in \mathbf{R}^{2}: p(x, y)=0\right\},
$$

considered as a subset of $\mathbf{R}^{2}$ in the usual topology. The Newton polygon of $p$ is defined to be the convex hull of the set

$$
N=\left\{(k, l) \in \mathbf{Z}^{2}: c_{k, l} \neq 0\right\} .
$$


If $E$ is an (oriented) edge of the Newton polygon with endpoints $k_{E}^{\prime}, l_{E}^{\prime}$ and $k_{E}^{\prime \prime}, l_{E}^{\prime \prime}$ then numbers $d_{E}, p_{E}$, and $q_{E}$ are defined by

$$
d_{E}=\operatorname{gcd}\left(k_{E}^{\prime \prime}-k_{E}^{\prime}, l_{E}^{\prime \prime}-l_{E}^{\prime}\right), \quad p_{E}=\frac{k_{E}^{\prime \prime}-k_{E}^{\prime}}{d_{E}}, \quad q_{E}=\frac{l_{E}^{\prime \prime}-l_{E}^{\prime}}{d_{E}},
$$

and the edge polynomial $e_{E} \in \mathbf{R}[t]$ by

$$
e_{E}(t)=\sum_{i=0}^{d_{E}} c_{k_{E}^{\prime}+i p_{E}, l_{E}^{\prime}+i q_{E}} t^{i} .
$$

An edge is called outer if it maximizes some linear function $a k+b l$ on the Newton polygon, where at least one of $a$ or $b$ is positive.

The purpose of this paper is to establish the following two theorems

Theorem 1 For the compactness of $C$ it suffices that

1. $p$ is not divisible by $x$ or $y$, and

2. the edge polynomials corresponding to outer edges have no real zeroes.

Theorem 2 For the compactness of $C$ it is necessary that

1. $p$ is not divisible by $x$ or $y$, and

2. the edge polynomials corresponding to outer edges have no real zeroes of odd order.

It is possible to give compactness criteria which are both necessary and sufficient, but these are much more complicated and require a knowledge of $c_{k, l}$ for $(k, l)$ in the interior of the Newton polygon, as the example

$$
p(x, y)=x^{8}-4 x^{6} y^{2}+6 x^{4} y^{4}-4 x^{2} y^{6}+y^{8}+c x^{2} y^{2}+1
$$

shows. Its zero set is easily seen to be compact if and only if $c \geq 0$, but the point $(2,2)$ lies inside the Newton polygon.

The edge polynomials are polynomials in a single variable, so the Sturm test 2 can be used to check for real zeroes.

It is, perhaps, of interest that Theorem 1 was developed for the single example

$$
\begin{aligned}
p(x, y)= & 72 x^{14}-\left(576+432 y^{2}\right) x^{13}+\left(1947+3552 y^{2}+1152 y^{4}\right) x^{12} \\
& -\left(3504+11988 y^{2}+10464 y^{4}+1440 y^{6}\right) x^{11} \\
& +\left(3452+20360 y^{2}+38762 y^{4}+15384 y^{6}+720 y^{8}\right) x^{10} \\
& -\left(1536+16456 y^{2}+71800 y^{4}+66316 y^{6}+10536 y^{8}\right) x^{9} \\
& +\left(2040 y^{2}+62966 y^{4}+143492 y^{6}+57803 y^{8}+2160 y^{10}\right) x^{8} \\
& -\left(-4608 y^{2}+8608 y^{4}+153832 y^{6}+154672 y^{8}+21648 y^{10}\right) x^{7} \\
& +\left(-20100 y^{4}+48272 y^{6}+208760 y^{8}+83120 y^{10}+2760 y^{12}\right) x^{6} \\
& -\left(-36120 y^{6}+104440 y^{8}+151552 y^{10}+20824 y^{12}\right) x^{5} \\
& +\left(-33769 y^{8}+109100 y^{10}+58958 y^{12}+1908 y^{14}\right) x^{4} \\
& -\left(-17900 y^{10}+62848 y^{12}+11680 y^{14}\right) x^{3} \\
& +\left(-5530 y^{12}+20912 y^{14}+944 y^{16}\right) x^{2} \\
& -\left(-972 y^{14}+3888 y^{16}\right) x+\left(-81 y^{16}+324 y^{18}\right)
\end{aligned}
$$


which arises in a problem on wave propagation on a singular solution of the Einstein-Maxwell equations in general relativity. See [4] for details.

\section{Sufficiency}

Assume that Theorem [1 is false, i.e. that for some $p$ satisfying the conditions there is a sequence of points $\left(x_{n}, y_{n}\right)$ such that

$$
p\left(x_{n}, y_{n}\right)=0
$$

with either $x_{n}$ or $y_{n}$ unbounded. Passing to a subsequence we may assume that either

$$
\left|x_{n}\right| \leq\left|y_{n}\right| \quad \text { and } \quad \lim _{n \rightarrow \infty}\left|y_{n}\right|=\infty
$$

or

$$
\left|y_{n}\right| \leq\left|x_{n}\right| \quad \text { and } \quad \lim _{n \rightarrow \infty}\left|x_{n}\right|=\infty .
$$

Without loss of generality we may assume the former. It then follows that

$$
a_{n}=\frac{\log \left|x_{n}\right|}{\log \left|y_{n}\right|} \leq 1
$$

Passing again to a subsequence, we may assume that this quantity either tends to a finite limit $a$ or that it tends to $-\infty$.

Supposing that $\lim _{n \rightarrow \infty} a_{n}=-\infty$, let

$$
L=\max _{(k, l) \in N, k=0} l
$$

and

$$
A=\min _{(k, l) \in N, k \neq 0} \frac{L-l}{k} .
$$

Then maximum in (12) is over a non-empty set since $x$ does not divide $p$. The minimum in (13) might be over an empty set. If it is not then, since $\lim _{n \rightarrow \infty} a_{n}=-\infty$, we may pass to a subsequence where

$$
a_{n} \leq A-1 .
$$

Then, for all $(k, l) \in N$ with $k \neq 0$,

$$
\frac{\log \left|x_{n}^{k} y_{n}^{l}\right|}{\log \left|y_{n}\right|}=k a_{n}+l \leq k a_{n}+L-k A \leq L-k \leq L-1
$$

so that

$$
\left|x_{n}^{k} y_{n}^{l}\right| \leq\left|y_{n}\right|^{L-1} .
$$

Passing to a subsequence where $\left|y_{n}\right|>1$, the same estimate holds for $(k, l) \in$ $N-(0, L)$ such that $k=0$. It now follows from the triangle inequality that

$$
\underset{(k, l) \in N-(0, L)}{\left|\sum_{k, l} x_{n}^{k} y_{n}^{l}\right| \leq\left|y_{n}\right|^{L-1}\left|\sum_{(k, l) \in N-(0, L)} c_{k, l}\right|}
$$


Once

$$
\left|y_{n}\right|>\left|\sum_{(k, l) \in N-(0, L)} c_{k, l}\right| /\left|c_{0, L}\right|
$$

the triangle inequality shows that

$$
\left|p\left(x_{n}, y_{n}\right)\right| \geq\left|c_{0, L}\right|\left|y_{n}\right|^{L}-\left|\sum_{(k, l) \in N-(0, L)} c_{k, l} x_{n}^{k} y_{n}^{l}\right|>0
$$

contradicting (8).

Suppose, then, that $\lim _{n \rightarrow \infty a_{n}}=a$. Define

$$
m=\max _{(k, l) \in N}(a k+l) .
$$

Let $M$ be the set where this maximum is taken. $M$ is either a vertex or an outer edge of the Newton polygon. In either case, define

$$
m^{\prime \prime \prime}=\max _{(k, l) \in N-M}(a k+l), \quad m^{\prime}=\frac{2 m+m^{\prime \prime \prime}}{3}, \quad m^{\prime \prime}=\frac{m+m^{\prime \prime}}{3} .
$$

It follows immediately that $m>m^{\prime}>m^{\prime \prime}>m^{\prime \prime \prime}$.

If $M$ is the single vertex $\left(k_{V}, l_{V}\right) \in N$ then

$$
\lim _{n \rightarrow \infty} \frac{\log \left|c_{k_{V}, l_{V}} x_{n}^{k_{V}} y_{n}^{l_{V}}\right|}{\log \left|y_{n}\right|}=a k_{V}+l_{V}=m
$$

so

$$
\log \left|c_{k_{V}, l_{V}} x_{n}^{k_{V}} y_{n}^{l_{V}}\right| \geq m^{\prime} \log \left|y_{n}\right|
$$

and hence

$$
\left|\sum_{(k, l) \in M} c_{k, l} x_{n}^{k} y_{n}^{l}\right| \geq\left|y_{n}\right|^{m^{\prime}}
$$

for all sufficiently large $n$.

If, on the other hand, $M$ is the outer edge $E$ with endpoints $\left(k_{E}^{\prime}, l_{E}^{\prime}\right)$ and $\left(k_{E}^{\prime \prime}, l_{E}^{\prime \prime}\right)$ then, since $e_{E}$ has no real zeroes, there is an $\epsilon_{E}>0$ such that

$$
\left|e_{E}(t)\right| \geq \epsilon_{E}
$$

for all $t$. Then

$$
\left|\sum_{(k, l) \in M} c_{k, l} x_{n}^{k} y_{n}^{l}\right|=\left|x_{n}^{k_{E}^{\prime}} y_{n}^{l_{E}^{\prime}} e_{E}\left(x_{n}^{p_{E}} y_{n}^{q_{E}}\right)\right| \geq \epsilon_{E}\left|x_{n}^{k_{E}^{\prime}} y_{n}^{l_{E}^{\prime}}\right| .
$$

It follows that

$$
\lim _{n \rightarrow \infty} \frac{\log \left|\sum_{(k, l) \in M} c_{k, l} x_{n}^{k} y_{n}^{l}\right|}{\log \left|y_{n}\right|}=a k_{E}^{\prime}+l_{E}^{\prime}=m
$$

and hence that (24) holds for sufficiently large $n$. 
In either case, for $(k, l) \in N-M$,

$$
\lim _{n \rightarrow \infty} \frac{\log \left|c_{k, l} x_{n}^{k} y_{n}^{l}\right|}{\log \left|y_{n}\right|}=a k+l \leq m^{\prime \prime \prime} .
$$

For sufficiently large $n$, we then have

$$
\left|c_{k, l} x_{n}^{k} y_{n}^{l}\right| \leq\left|y_{n}\right|^{m^{\prime \prime}}
$$

and hence

$$
\left|\sum_{(k, l) \in N-M} c_{k, l} x_{n}^{k} y_{n}^{l}\right| \leq \#(N-M)\left|y_{n}\right|^{m^{\prime \prime}} .
$$

For $n$ sufficiently large,

$$
\left|y_{n}\right|^{m^{\prime}}>\#(N-M)\left|y_{n}\right|^{m^{\prime \prime}}
$$

and hence, by the triangle inequality,

$$
\left|p\left(x_{n}, y_{n}\right)\right| \geq\left|\sum_{(k, l) \in M} c_{k, l} x_{n}^{k} y_{n}^{l}\right|-\left|\sum_{(k, l) \in N-M} c_{k, l} x_{n}^{k} y_{n}^{l}\right|>0,
$$

contradicting (8).

\section{$3 \quad$ Necessity}

Condition 1 is obviously necessary for compactness. Suppose condition 2 is violated. Then there is some outer edge $E$ with endpoints $\left(k_{E}^{\prime}, l_{E}^{\prime}\right)$ and $\left(k_{E}^{\prime \prime}, l_{E}^{\prime \prime}\right)$ for which $e_{E}$ has a zero of odd order. Since $e_{E}(0)=c_{k_{E}^{\prime}, l_{E}^{\prime}}$ and $\left(k_{E}^{\prime}, l_{E}^{\prime}\right)$ is an extreme point of the Newton polygon we know that $e_{E}(0) \neq 0$. On either side of a zero of odd order there are points $t_{+}$and $t_{-}$where

$$
e_{E}\left(t_{+}\right)>0 \quad \text { and } \quad e_{E}\left(t_{-}\right)<0 .
$$

We may take $t_{+}$and $t_{-}$to have the same sign. Since $E$ is an outer edge there are $a$ and $b$, at least one of which is positive, such that

$$
a k+b l
$$

is maximized for $(k, l) \in E$. Let $m$ be this maximum and

$$
m^{\prime \prime \prime}=\max _{(k, l) \in N-E}(a k+b l)
$$

Since

$$
\operatorname{gcd}\left(p_{E}, q_{E}\right)=1
$$

there are integers $r$ and $s$ such that

$$
r p_{E}+s q_{E}=1 .
$$


Setting

$$
x_{n}(t)=t^{r} n^{a} \quad \text { and } \quad y_{n}(t)=t^{s} n^{b}
$$

we see that

$$
x_{n}(t)^{k} y_{n}(t)^{l}=t^{r k+s l} n^{a k+b l} .
$$

If $k=k_{E}^{\prime}+i p_{E}$ and $l=l_{E}^{\prime}+i q_{E}$ then

$$
x_{n}(t)^{k} y_{n}(t)^{l}=t^{r k_{E}^{\prime}+s l_{E}^{\prime}+i} n^{m} .
$$

From this it follows that

$$
\sum_{(k, l) \in E} c_{k, l} x_{n}(t)^{k} y_{n}(t)^{l}=t^{r k_{E}^{\prime}+s l_{E}^{\prime}} n^{m} e_{E}(t) .
$$

If $(k, l) \in N-E$ then

$$
\sum_{(k, l) \in N-E} c_{k, l} x_{n}\left(t_{+}\right)^{k} y_{n}\left(t_{+}\right)^{l}=\sum_{(k, l) \in N-E} c_{k, l} t_{+}^{r k+s l} n^{a k+b l}
$$

and hence, by the triangle inequality,

$$
\left|\sum_{(k, l) \in N-E} c_{k, l} x_{n}\left(t_{+}\right)^{k} y_{n}\left(t_{+}\right)^{l}\right| \leq \sum_{(k, l) \in N-E} c_{k, l} t_{+}^{r k+s l} \mid n^{m^{\prime \prime \prime}}
$$

If $n$ is sufficiently large that

$$
t^{r k_{E}^{\prime}+s l_{E}^{\prime}} e_{E}\left(t_{+}\right) n^{m}>\sum_{(k, l) \in N-E} c_{k, l} t_{+}^{r k+s l} \mid n^{m^{\prime \prime \prime}}
$$

then another application of the triangle inequality shows that

$$
p\left(x_{n}\left(t_{+}\right), y_{n}\left(t_{+}\right)\right)
$$

has the same sign as

$$
t_{+}^{r k_{E}^{\prime}+s l_{E}^{\prime}}
$$

Similarly, for large $n$,

$$
p\left(x_{n}\left(t_{-}\right), y_{n}\left(t_{-}\right)\right)
$$

has the opposite sign from

$$
t_{-}^{r k_{E}^{\prime}+s l_{E}^{\prime}}
$$

and hence $p\left(x_{n}\left(t_{+}\right), y_{n}\left(t_{+}\right)\right)$and $p\left(x_{n}\left(t_{-}\right), y_{n}\left(t_{-}\right)\right)$are of opposite sign. By the intermediate value theorem there is then a $t_{n}$ between $t_{-}$and $t_{+}$for which

$$
p\left(x_{n}\left(t_{n}\right), y_{n}\left(t_{n}\right)\right)=0 .
$$

Since at least one of $a$ or $b$ is positive at least one of $x_{n}\left(t_{n}\right)$ or $y_{n}\left(t_{n}\right)$ is unbounded as $n$ tends to infinity. The curve $C$ is therefore not compact. 


\section{References}

[1] Maria Jesus de la Puente. Real plane algebraic curves. Expo. Math., 20(4):291-314, 2002.

[2] L.E. Dickson. First Course in the Theory of Equations. John Wiley \& Sons, New York, 1922.

[3] Dominique Duval. Rational Puiseux expansions. Compositio Math., 70(2):119-154, 1989.

[4] John Stalker and Abdolreza Shadi Tahvildar-Zadeh. Scalar waves on a naked-singularity background. 2003. 